\title{
ALFABETIZACIÓN DIGITAL: UN PELDAÑO HACIA LA SOCIEDAD DE LA INFORMACIÓN
}

\author{
MYRNA CAROLINA MARTI*, MARCELO JOSÉ D'AGOSTINO**, \\ JORGE VEIGA DE CABO $* * *$, JAVIER SANZ-VALERO****, *****
}

(*) Psicóloga especializada en Tecnologías de Información y consultora externa del Área de Información y Gestión del Conocimiento. Organización Panamericana de la Salud, Oficina Regional de la Organización Mundial de la Salud (OPS/OMS). Washington DC. EEUU.

(**) Área de Información y Gestión del Conocimiento. Organización Panamericana de la Salud, Oficina Regional de la Organización Mundial de la Salud (OPS/OMS). Washington DC. EEUU.

(***) Escuela Nacional de Medicina del Trabajo. Instituto de Salud Carlos III. Madrid. España.

(*****) Departamento de Enfermería Comunitária, Medicina Preventiva y Salud Pública e

Historia de la Ciencia, Universidad de Alicante. Alicante. España.

(*****) Departamento de Salud Pública, Historia de la Ciencia y Ginecología, Universidad Miguel Hernández. Elche. España

\section{RESUMEN}

Los seres humanos tienden a reaccionar ante las palabras por sus antónimos. Lo primero que se piensa cuando se habla de alfabetización es en los analfabetos. Lamentablemente esta palabra tiene un antónimo muy fuerte y con implicancias sociales y culturales muy negativas. Esta es una de las grandes críticas a la tendencia de llamar "alfabetización" a esta renovación continua de las capacidades de aprender y comunicarse. En este articulo coincidimos con los pensadores que sostienen que la palabra "alfabetización, significando la capacidad para leer y escribir, ha extendido gradualmente su campo de aplicación en la era digital para pasar a significar la capacidad para utilizar las tecnologías de información y comunicaciones y entender la información, cualquiera que sea su formato. Presentamos a la alfabetización como un proceso constante y permanente de aprendizaje que debe perdurar por toda la vida de las personas para que las mismas sean funcionales en su vida en familia, en sociedad y en las organizaciones en las que trabajan en este nuevo paradigma llamado "Sociedad de la Información". La Alfabetización Digital es el último peldaño conocido de esta escalada hacia la Sociedad de la Información. Estamos en el medio de un proceso de transformación permanente que nos impone repensar nuestras relaciones con la realidad y debemos estar preparados para actuar en consecuencia ya que el tiempo y la disponibilidad y el acceso a información se han transformado en elementos claves de supervivencia en esta nueva Sociedad. .

\section{PALABRAS CLAVES}

Alfabetización digital, Sociedad de la Información, Acceso a información, Derechos humanos, Tecnologías de Información y Comunicaciones.

\section{ABSTRACT}

Human beings tend to react to the words by their antonymous. The first thing that comes to the mind when speaking of literacy is in the illiterate. Unfortunately, this word has a very strong antonymous and with highly negative social and cultural implications. This is one of the great criticisms to the tendency to call "literacy" to this ongoing renewal of the capacities of learning and communicating. In this article we agree with those thinkers who state that the word "literacy", meaning the ability to read and write, has gradually extended its scope of application in the digital era to move to mean the ability to use the information and communications technologies and to understand the information, whatever its format. We present literacy as a constant and permanent learning process which should continue during life so that people can be functional for their family life, for the society and for the organizations in which they work, in this new paradigm called "Information Society". Digital Literacy is the last step known in this escalation towards the Information Society. We are in the middle of a permanent process of transformation which compels us to rethink our relationship with reality and we must be prepared to act accordingly since time and availability and the access to information have become key elements of survival in this new Society.

\section{KEY WORDS}

Digital Literacy, Information Society, Information Access, Human Rights, Information and Communication Technologies. 


\section{ANTECEDENTES}

El concepto «Alfabetización Digital» se comenzó a utilizar en los años 90 para designar al conjunto de destrezas, conocimientos y actitudes que necesita una persona para poder desenvolverse funcionalmente dentro de la Sociedad de la Información. Se diferencia sustancialmente de la Alfabetización Informacional porque se agrega el componente de "construcción del conocimiento", con evidencia a través de múltiples fuentes y no sólo de Internet.

Por tanto, se ha producido un giro sustancial en el significado del concepto «Alfabetización», llevándolo más allá de la pura capacidad de leer y escribir y adaptándolo a las nuevas necesidades provenientes de la Sociedad de la Información, Definimos a la alfabetización como la posesión de destrezas que se necesitan para conectarse a la información imprescindible para sobrevivir en socie$\operatorname{dad}^{1,2}$.

En consecuencia, este concepto se ha complementado con las llamadas con las llamadas «Alfabetizaciones en destrezas», conceptos desarrollados para hacer referencia a una información de creciente complejidad y a las tecnologías en auge y su implicación en el mundo laboral ${ }^{3,4}$.

La UNESCO es contundente en su recomendación a los países en cuanto a priorizar la alfabetización digital en todos los niveles de la sociedad. Es por eso que los países, conjuntamente con programas de gobiernos digitales, están llevando a cabo proyectos de «alfabetización digital», dirigidos a los ciudadanos. Las Instituciones y personas no pueden permanecer alejadas de esta tendencia de los gobiernos y deben tomar esto como una obligación de todos, entendiendo que la Alfabetización Digital no debe ser vista como una necesidad simplemente, sino que debe ser vista como un derecho inherente de las personas.

Para esta Organización, la tradicional definición de alfabetización que se enfocaba en el desarrollo de las capacidades de lecto-escritura y conocimientos de aritmética, ha sido expandida a una nueva definición que incluye conocimientos de computación y capacidad de adquirir información. O sea, la capacidad de tomar decisiones, crear valores, resolver problemas y colaborar con otros por medio del uso de las tecnologías electrónicas y de las redes5.

La tendencia actual ha llevado a los expertos en el tema a agrupar las alfabetizaciones por destrezas, dentro de este marco encontramos diferentes con- ceptos de aparición cronológica que pueden considerarse los predecesores de la alfabetización digital:

1. Alfabetización Bibliotecaria: Término surgido a principio de los años 80 , cuyo objetivo era lograr que «el usuario que la posea pueda seguir un camino o estrategia de búsqueda sistemáticos para localizar un texto y evaluar la relevancia de la información ${ }^{6}$.

2. Alfabetización de medios: Usuarios capaces de responder con una actitud crítica en la evaluación de la información obtenida a través de los medios de comunicación de masas: televisión, diarios, revistas, radio e Internet. Su objetivo principal es que la gente pueda «comprender, producir y negociar significados, en una cultura hecha de imágenes, palabras y sonidos poderosos», al alcanzar la competencia en medios una persona puede «decodificar, evaluar, analizar y producir medios, tanto impresos como electrónicos» ${ }^{7}$.

3. Alfabetización informática o en Tecnologías de la información y el Conocimiento (TIC): Usuarios que poseen las destrezas prácticas en tecnologías de la información necesarias para el trabajo y para la vida diaria. Son aquellas destrezas necesarias para poner en marcha un paquete de aplicaciones informáticas: procesadores de texto, planillas de cálculo, bases de datos, etc, junto con algunas destrezas generales propias de TIC: grabar documentos, archivar, utilizar disketes, CDs, Pen Drives, imprimir, etc. En resumen es la capacidad de poder utilizar un ordenador correctamente, como una herramienta eficaz;

4. Alfabetización Informacional: Esta alfabetización en un plano más evolucionado que las anteriores pone el énfasis en la resolución de problemas haciendo uso eficaz de la información.

En el presente debe considerarse que todas las alfabetizaciones antes mencionadas, forman parte de la Alfabetización Digital, este concepto las engloba y juntas se potencian para llegar a una alfabetización completa y necesaria en la Sociedad de la Información. Según Glister «La alfabetización digital tiene que ver con el dominio del mundo de las ideas, no de las teclas» ${ }^{8}$.

\section{¿CUÁLES SON LOS OBJETIVOS DE LA ALFABETIZACIÓN DIGITAL?}

- Desarrollar capacidades para la construcción e implementación de un programa personal y/o Institucional de renovación constante del 
saber en el uso de las Tecnologías de Información y Comunicación aplicadas, así como en metodologías para acceso a información y gestión del conocimiento.

- Entregar herramientas y metodologías que permitan entender el proceso de conversión del saber tácito en explícito y funcional.

- Desarrollar capacidades para la aplicación de los nuevos saberes, considerando el impacto psicológico en la adopción de las nuevas tecnologías de información y comunicación en el día a día de las personas.

- Reforzar las competencias individuales e institucionales en Tecnologías de información y Comunicación aplicadas, así como en metodologías para acceso a información y gestión del conocimiento, de forma tal que puedan afrontar y acompañar el cambio organizacional y de vida frente a la Sociedad de la Información.

- Adquirir nuevos hábitos de trabajo frente a a tres grandes cambios:

- Nuevos tipos de documentos.

- Nuevas formas de comunicar.

- Nuevos entornos de comunicación y educación.

- Conservación y recirculación del conocimiento generado en nuevas fuentes de información basadas en evidencias y lecciones aprendidas.

- Aplicación del conocimiento con innovación para la solución de situaciones novedosas, emergentes y/o desconocidas.

- Aplicación eficaz y eficiente del saber en un proceso de mejora continua de las actividades rutinarias.

- Adquirir confianza y un dominio completo de los conceptos fundamentales para el uso de recursos tecnológicos y metodológicos.

- Adquirir nuevos hábitos de trabajo en los que preponderan:

- la capacidad analítica de situaciones complejas.
- la identificación, análisis y solución de problemas.

- la planificación.

- la organización.

- la evaluación crítica de las situaciones de trabajo extraordinarias.

\section{¿CUÁLES SON LAS DESTREZAS \\ NECESARIAS PARA QUE UN INDIVIDUO SE CONSIDERE ALFABETIZADO DIGITAL?}

- Dominar el "arte del pensamiento crítico", realizando juicios de valor informados y equilibrados distinguiendo entre el contenido y su presentación.

- Lectura, escritura y comprensión en un contexto de hipertexto dinámico y no secuencial.

- Construcción de conocimiento: construir un conjunto de información fiable proveniente de diversas fuentes, con la capacidad de recoger y evaluar tanto el hecho como la opinión, en lo posible con objetividad.

- Habilidades de búsqueda utilizando el concepto de los motores de búsqueda de Internet.

- Creación de una "estrategia personal de información", que incluye selección de fuentes, mecanismos de distribución y entendimiento de nuevas tecnologías que facilitan el acceso a múltiples fuentes de información sin interacción manual.

- Participación activa en comunidades virtuales con el fin de contactarse con otra gente, debatir temas, compartir lecciones aprendidas y saber pedir ayuda.

- Capacidad para comprender un problema y seguir pasos para resolverlo.

- Capacidad de aprender: saber cómo se organiza el conocimiento, cómo encontrar la información y cómo utilizar la información de forma que otros puedan aprender (recirculación del conocimiento).

\section{ALGUNOS EJEMPLOS EN PAÍSES DE LAS AMÉRICAS}

Dentro del marco Educación para Todos, la 
UNESCO recomienda la formación basada en tecnologías de la Información para todos los ciudadanos y propone al ámbito laboral para reforzar la alfabetización básica de los ciudadanos basándola en competencias para poder desenvolverse en la era de la información.

En respuesta a las recomendaciones de UNES$\mathrm{CO}$, se están implementando en algunos países varios proyectos de alfabetización digital. Los mismos están orientados a la formación de los ciudadanos en competencias necesarias para que puedan desenvolverse funcionalmente en la Sociedad de la Información.

Argentina: proyecto educ.ar, dirigido a docentes y estudiantes, con esta iniciativa se busca instalar en el sistema educativo el uso de la tecnología en el aula y lograr un uso eficiente del equipamiento recibido y existente, como así también reforzar el vínculo escuela-universidad.

Chile: la Campaña Nacional de Alfabetización Digital, que se desarrollará en el período 2003-2005, tiene como objetivo capacitar a medio millón de chilenos mayores de 15 años que están fuera del sistema escolar para que obtengan formación práctica en el uso de las Tecnologías de Información y Comunicación (TIC). Se trata de preparar a la ciudadanía para vivir en una sociedad globalizada y aprovechar las oportunidades que ofrecen las nuevas tecnologías digitales. La iniciativa está dirigida a vastos sectores de la población que hasta hoy no han tenido acceso a la computación e Internet, especialmente trabajadores, microempresarios y madres.

Brasil: dentro del programa Alfabetización Solidaria, se lanzó el proyecto alfabetización digital, comenzando por 20 ciudades del nordeste brasilero, siendo sus principales objetivos:

- Mantener un trabajo continuo entre la Instituciones aparcera del municipio beneficiado, principalmente entre el coordinador del proyecto Alfabetización Digital de Instituciones de Enseñanza Superior, el agente local del municipio y los alfabetizadores.

- Promover la capacitación continuada del agente local y de los alfabetizadores.

- Contribuir a la democratización de la informática.

- Proporcionar a la comunidad, a los alfabetizadores y a los alumnos egresos de la Alfa- betización Solidaria, el conocimiento de informática y de herramientas de Internet, así como difundir el uso del computador como instrumento auxiliar en las actividades cotidianas y profesionales.

Perú: Con la finalidad de fomentar el uso de los medios informáticos en el departamento de Miraflores, la comuna de este distrito está promoviendo iniciativas educativas digitales en diversos centros educativos; es decir, creando espacios de alfabetización digital, que permitan en un futuro cercano optimizar más aún la relación vecinos- municipio. Esta nueva propuesta de aprendizaje virtual denominada MIRA RED, que se sustenta en la etapa escolar, se proyecta a todos los vecinos con el objetivo de que la población se adecue en el manejo de la tecnología digital.

\section{TRES MENSAJES IMPORTANTES PARA DESTACAR}

- Las organizaciones, países y sociedades que Ignoren la necesidad de mejorar la alfabetización digital no estarán en situación de competir de manera eficaz en la nueva era de la información ${ }^{9}$.

- Estamos en el medio de un proceso de transformación permanente que nos impone repensar nuestras relaciones con la realidad y debemos estar preparados para actuar en consecuencia, ya que el tiempo, la disponibilidad y el acceso a información se han transformado en elementos claves de supervivencia y desarrollo personal en esta nueva Sociedad de la Información ${ }^{10}$.

- Acceder al conocimiento correcto en el momento oportuno, debe dejar de ser una prerrogativa de pocos y pasar a ser in derecho inherente de las personas que viven en socie$\operatorname{dad}^{11,12}$.

\section{A MODO DE REFLEXIÓN FINAL}

Vivimos en un mundo donde se puede acceder a documentación, de mayor o menor calidad a través de múltiples caminos. Sin embargo, tener la formación para conocer su verdadero valor y aptitud es un privilegio que no está al alcance de todas las personas $^{13,14}$.

De igual modo, para cualquier trabajador, en el maremágnum de información que manejan hoy en día las empresas, dominar los métodos y las herra- 
mientas que sirven para discernir lo que es realmente útil se ha convertido en una de las habilidades más demandadas en el mundo laboral, esta precariedad

\section{BIBLIOGRAFIA}

1. Silvera C. La alfabetización digital: una herramienta para alcanzar el desarrollo y la equidad en los países de América Latina y el Caribe. ACIMED [revista en Internet]. 2005 [citado 22 feb 2008];13(1): [aprox. 15 pantallas]. Disponible en:

http://bvs.sld.cu/revistas/aci/13_1_05/aci04105.pdf

2. Comisión Económica para América Latina y el Caribe (CEPAL). Los caminos hacia una sociedad de la información en América Latina y el Caribe. Santiago de Chile: Organización de las Naciones Unidas;2003.

3. Bawden D. Revisión de los conceptos de alfabetización informacional y alfabetización digital. An Document. 2002;5:361-408.

4. Royal Society of Arts (RSA). RSA computer literacy and information technology. $2^{\mathrm{a}}$ ed. Oxford: Heinemann Educational; 1993.

5. Organización de las Naciones Unidas para la Educación, la Ciencia y la Cultura (UNESCO). Proyecto regional de educación para América Latina y el Caribe. (PRELAC) [monografía en Internet]. La Habana, Cuba: UNESCO; 2002 [citado 22 feb 2008]. Disponible en: http://www.unesco.cl/medios/biblioteca/documentos/prelac_proyecto_regional_educacion_esp.pdf

6. Fatzer JB. Library literacy. RQ. 1987;26(3):313-4.

7. Aufderheide P, Firestone CM. Media literacy: a report of the national leadership conference on media literacy. Washington DC: The Aspen Institute; 1993. del exceso contrasta con la frágil formación de una gran mayoría de personas ${ }^{15}$.

8. Gilster P. Digital literacy. New York NY: Wiley; 1997.

9. Oxbrow N. Employment tends for information professionals. In: Oppenheim C, Citroen CL, Griffiths JM. Perspectives in Information Management vol 2. London: Bowker-Saur; 1990. p. 143-156.

10. McGarry K. The chainging context of information. $2^{\circ}$ ed. London: Library Association Publishing; 1993.

11. Asamblea General de las Naciones Unidas. Declaración Universal de los Derechos Humanos, artículo 27.1. Ginebra: Organización de las Naciones Unidas; 1948.

12. Siles Rojas C, Reyes Rebollo MM. Formación a través de las nuevas tecnologías para el fomento de una sociedad más democrática. Revista Electrónica de Tecnología Educativa (Edutec) [revista en Internet]. 2003 [citado 22 feb 2008];16: [aprox. 11 pantallas]. Disponible en: http://www.uib.es/depart/gte/edutec-e/revelec16/siles.htm

13. Sanz-Valero J, Veiga de Cabo J, Castiel LD. A iniciativa Open Access no acesso à informação técnicocientífica nas Ciências da Saúde. RECIIS R Eletr Com Inf Inov Saúde. 2007;1(1):19-26.

14. Sanz-Valero J, D’Agostino MJ, Castiel LD, Veiga de Cabo J. La iniciativa Open Access, una visión de conjunto. Med Segur Trab. 2007;53(207):5-10.

15. Castiel LD, Vasconcellos-Silva PR. Precariedades do Excesso: informação e comunicação em saúde colectiva. Río de Janeiro: Editor Fiocruz; 2006. 
